\title{
Helical Electronic Transitions of Spiroconjugated Molecules
}

\author{
Marc H. Garner, and Clemence Corminboeuf*
}

Laboratory for Computational Molecular Design, Institute of Chemical Sciences and Engineering, Ecole Polytechnique Federale de Lausanne (EPFL), 1015 Lausanne, Switzerland.

Supporting Information Placeholder

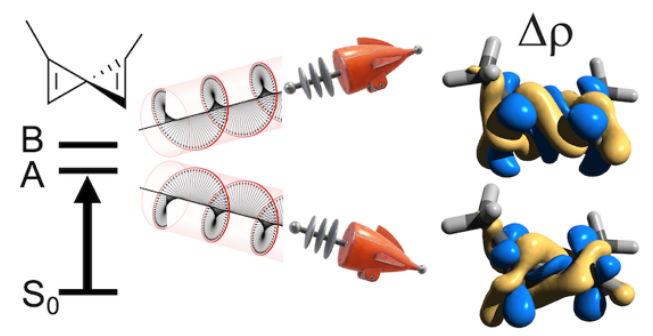

\begin{abstract}
The two perpendicularly oriented $\pi$-systems of allene mix into helical molecular orbitals (MOs) when the symmetry of the molecule is reduced. However, the $\pi-\pi^{*}$ transitions of allenes are linear combinations of two excitations that always consist of both helicities; consequently, the electronic transitions are not helical. Here, we examine the electronic structure of spiroconjugated molecules, which have the same parent symmetry as allene but with different relative orientation of the two $\pi$-systems. We show how the $\pi$-mixing in spiropentadiene is analogous to the helical $\pi$-mixing in allene. However, in spiroconjugated systems only half the $\pi$ MOs become helical. Due to this difference, the $\pi-\pi^{*}$ transitions in substituted spiropentadiene come in near-degenerate pairs where the helicity is symmetry protected, and consequently there is no significant mixing between excitations involving MOs of opposite helicity. This inherent helicity of the $\pi-\pi^{*}$ transitions is verified by computation of the change of electron density. These transitions have big rotatory strengths where the sign correlates with the helicity of the transition. The electronic helicity of spiroconjugated molecules thus manifests itself in observable electronic and optical properties.
\end{abstract}

Our chemical intuition is often challenged because one simple electronic structure model cannot perfectly account for every type of molecular property. ${ }^{1}$ The electrohelicity effect that has been described in chiral allenes, as well as longer cumulenes and polyynes, is a particular fascinating and ambiguous case. ${ }^{2}$ In these linear molecules, their otherwise orthogonal $\pi$-systems can mix and form helical molecular orbitals (MOs). ${ }^{2-}$ ${ }^{4}$ However, an MO is not an observable property of a molecule. But although purely a theoretical construct for the purpose of computing properties of molecules, MOs have successfully provided insight into the fundamental nature of electrons in molecules, ${ }^{5}$ most famously the selection rules for pericyclic reactions. ${ }^{6-9}$ The understanding of helical $\pi$-conjugation through MOs may provide new chemical intuition. ${ }^{2}$ However, while the molecules where the effect appear are single-handed chiral, both helicities are present in the electronic structure. The chirality associated with the electronic structure is thus more complex than that of the of molecular structure. ${ }^{10}$ This dual-helicity may limit the possibility of experimentally observable effects arising from the helicity of any specific MO, but some relations to properties have been proposed in recent years. ${ }^{2,11-20}$

The helicity of the electronic structure in allene (Scheme 1, left) is mediated by the $p$-orbitals on its central $s p$-hybridized carbon. As discussed in detail in prior publications, an initial choice is made for the orientation of these two $p$-basis functions. ${ }^{4,21}$ By convention, we orient allene relative to its two mirror-planes. Shown in Figure 1a, the $p_{x}$ and $p_{y}$ basis functions are thereby symmetry-adapted to the sub-groups of the $D_{2 d}$ point group that have mirror-plane symmetry, the most notable being $C_{2 v}$. However, the $p_{x}$ and $p_{y}$ basis functions can be rotated $45^{\circ}$ around the allenic axis by forming linear combinations of the two, thus making a coarctate orbital system; i.e., one where there is a continuous $\pi$-overlap between the carbon termini despite their relative orientation of $90^{\circ} .^{4,22,23}$ Shown in the right column of Figure 1a, this basis is symmetry-adapted to the subgroups of $D_{2 d}$ that retain its rotation axes, such as $D_{2}$. These two bases are connected by a unitary transformation and the choice between them is arbitrary. ., $21^{2}$

\section{Scheme 1. Allene and Spiro-type Molecules.}

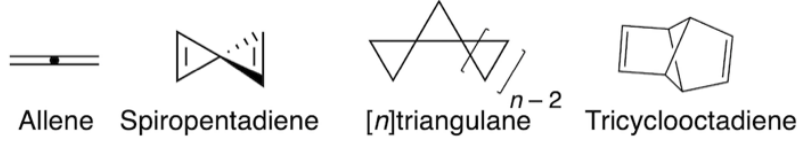

In spiroconjugated molecules, two $\pi$-systems are separated by an $s p^{3}$-hybridized carbon atom. ${ }^{24,25}$ The two $\pi$-systems are oriented $90^{\circ}$ relative to each other and interact by a well-described through-space interaction. ${ }^{26}$ Spiropentadiene is the simplest spiroconjugated molecule, constituting two ethene units fused together through a fifth spiro-carbon as shown in Scheme 1. Similar to allene, the parent spiropentadiene has $D_{2 d}$ symmetry with the same number of $\pi$-electrons. ${ }^{27}$ Shown in Figure $1 \mathrm{~b}$, we can make an analogous basis set rotation of the $p$-basis 
functions on the central carbon atom of spiropentadiene. These $p$-orbitals on the formally saturated spiro-carbon will participate in the $\sigma$-bonds of the molecule and are normally considered in the form of $s p^{x}$ hybrid orbitals; however, the $p$-orbitals, which mathematically are part of the carbon basis set, can mediate the possible through-bond coupling between the two $\pi$-systems. This through-bond effect has been described in the related saturated triangulanes (Scheme 1), where helical $\sigma$-orbitals appear along the full length of the molecular backbone. ${ }^{28,29}$ The $p$-orbitals in Figure $1 \mathrm{~b}$ are available as relay orbitals on the spirocarbon, and is equivalent to the way $\sigma$-orbitals mediate throughbond coupling between the $\pi$-systems in larger tricyclic analogues of spiroconjugated molecules (Scheme 1) as described by Gleiter and co-workers. ${ }^{30,31}$ While this is conceptually similar to allene, the two $\pi$-systems are oriented different spatially in spiropentadiene. As we shall see, this difference has a profound effect on the electronic structure and $\pi-\pi^{*}$ transitions.

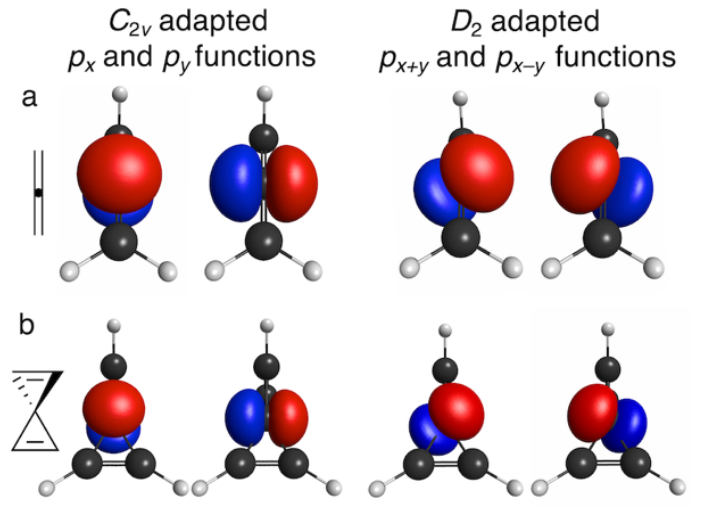

Figure 1. Basis functions of the central carbon atom symmetryadapted to the $C_{2 v}$ and $D_{2}$ point groups, which are both sub-groups of the full $D_{2 d}$ point group of allene (a) and spiropentadiene (b).

This letter proceeds as follows. We revisit the electronic structure and $\pi-\pi^{*}$ transitions of allene and its 1,3-disubstituted derivates. 1,3-disubstituted allene constitutes the simplest case of a molecule with helical MOs, and we show how the symmetry of the parent allene dictates that their electronic transitions cannot exhibit clear helicity. In contrast, we demonstrate that in spiroconjugated molecules both the electronic structure and $\pi-\pi^{*}$ transitions have distinct helicity associated to them because the helicity is symmetry-protected. These transitions show helical change of electron density and have high rotatory strengths where the sign depends on the helicity of the transition. Computations are carried out using density functional theory (DFT) as implemented in Gaussian $16 .{ }^{32}$ Excited states and optical properties are computed using time-dependent DFT. ${ }^{33-35}$

Like the longer cumulenes and polyynes, allene is routinely described as having two orthogonal $\pi$-systems lying in each their plane. ${ }^{36,} 37$ The highest occupied MO (HOMO) and HOMO- 1 are degenerate $\pi$-orbitals as shown in Figure 2. However, when the symmetry of the molecule is reduced from $D_{2 d}$ to $C_{2}$ by substituting two of the hydrogens, the $\pi$-systems mix and helical $\pi$-MOs are formed. ${ }^{2,4}$ In the case of $R$-1,3-dimethyallene the HOMO is an $M$-helix and the HOMO- 1 is a $P$ helix. In $D_{2 d}$-allene, the resulting $\pi \rightarrow \pi^{*}$ transitions between the two degenerate occupied and two degenerate unoccupied $\pi$ MOs consist of the four possible linear combinations of excitations between the two sets of MOs. As outlined in Figure 3, the four transitions are non-degenerate and belong to $A_{2}, B_{1}, A_{1}$, and $\mathrm{B}_{2}$ irreducible representations. ${ }^{38}$ Of these four, only the $\mathrm{B}_{2}$ transition is electric-dipole allowed, and it turns out to be the $\mathrm{S}_{0} \rightarrow \mathrm{S}_{4}$ transition. Reducing the symmetry of allene by substituents does not change much and the first three transitions remain quasi-forbidden in dimethylallene. The optical properties of substituted allenes have been explored experimentally in great detail. ${ }^{39-44}$ The transitions are all linear combinations of the possible excitations between the occupied $\pi_{P / M}$ and unoccupied $\pi_{P / M^{*}}$, with almost equal weighting. Oscillator strengths and magnitude of the MO weights will of course be method dependent to some extent; a qualitatively similar result is achieved at the CASPT2 $(8,8)$ /cc-pvdz level (Figure S1).

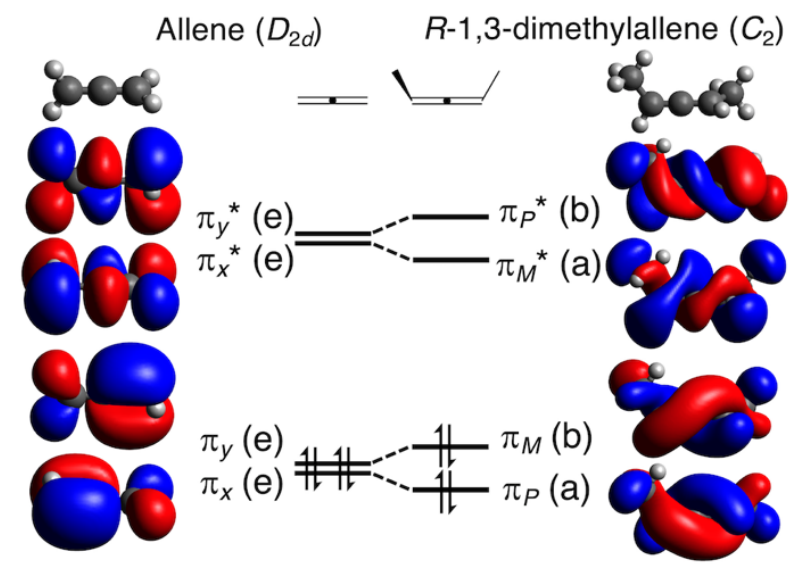

Figure 2. HOMO-1 to LUMO+1 of allene and R-1,3-dimethylallene computed at the $\omega$ B97X-D/Def2-TZVP level of theory. ${ }^{45,46}$

Figure $3 \mathrm{~b}$ shows the change of electron density during the $\pi \rightarrow \pi^{*}$ transitions of $R-1,3$-dimethylallene. Although chiral features appear, no helicity is apparent in the change of density. We attribute this lack of helicity to the transitions being superpositions of helical MO excitations, which we show schematically in Figure S4. While any individual excitation between helical MOs yields a helical pattern in the change of electron density, transitions between their linear combinations show a linear pattern. The helical HOMO and HOMO-1 can be split energetically by substituents. ${ }^{15,47}$ This splitting enhances the chiroptical response mediated by the strong $\mathrm{S}_{0} \rightarrow \mathrm{S}_{4}$ transition, ${ }^{20}$ which has notable rotatory strength in dimethylallene as listed in Figure $3 \mathrm{~b}$. Still, the response is limited by the near-equal contributions of $\pi_{M} \rightarrow \pi_{M}{ }^{*}$ and $\pi_{P} \rightarrow \pi_{P}{ }^{*}$. Even with increased energetic splitting of the helical MO pairs, the electronic transitions of allene do not become helical. ${ }^{20}$

In spiroconjugated molecules the two $\pi$-systems mix through-space. Still, the $\pi$-electrons cannot be considered fully shared between the two rings in carbon-based spiroconjugated systems. ${ }^{48-50}$ The simplest one, spiropentadiene, is a fairly unstable motif due to bond strain, ${ }^{51}$ but has nonetheless been synthesized with some variation. ${ }^{52-56}$ Shown in the left column of Figure 4a, its HOMO and HOMO-1 are degenerate and each have clear $\pi$-character on one side of the molecule. The LUMO and $\mathrm{LUMO}+1$ are non-degenerate and the two $\pi$-segments mix due to through-space $\pi$-overlap, which is characteristic of spiroconjugated systems. ${ }^{24-26}$ Shown in the right column of Figure 4 , the disubstitution we performed on allene has a similar effect on spiropentadiene. The symmetry is reduced from $D_{2 d}$ to $C_{2}$ and the degenerate $\pi_{x}$ and $\pi_{y}$ mix through-bond via the central 
carbon atom into helical $\pi_{P}$ and $\pi_{M}$. The unoccupied $\pi_{x+y}{ }^{*}$ and $\pi_{x-y} *$ are largely unchanged and do not mix further.

a

$$
\begin{aligned}
& \text { Point group: } \boldsymbol{D}_{2 \boldsymbol{d}} \\
& \overline{\mu_{\mathrm{x}, \mathrm{y}, \mathrm{z}}}=\mathrm{E}, \mathrm{E}, \mathrm{B}_{2} \\
& \mathrm{E} \times \mathrm{E}=\mathrm{A}_{1}, \mathrm{~A}_{2}, \mathrm{~B}_{1}, \mathrm{~B}_{2}
\end{aligned}
$$

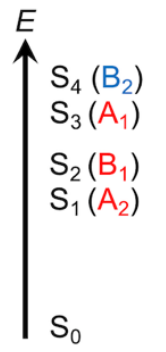

$$
\begin{aligned}
& \text { Point group: } C_{2} \\
& \mu_{x, y, z}=B, B, A \\
& A \times A=A \\
& A \times B=B \\
& B \times B=A
\end{aligned}
$$

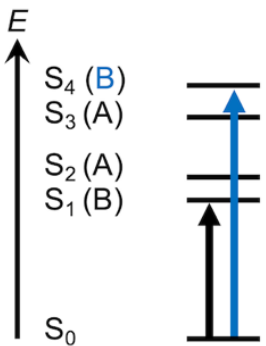

b

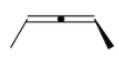

$\mathbf{S}_{0} \rightarrow \mathbf{S}_{4} \quad f=1.253 \quad R=55.4$

$0.50 \cdot\left(\pi_{M} \rightarrow \pi_{M}{ }^{*}\right)-0.45 \cdot\left(\pi_{P} \rightarrow \pi_{P}^{*}\right)$

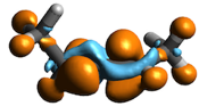

$$
\begin{aligned}
& \mathbf{S}_{0} \rightarrow \mathbf{S}_{3} \quad f=0.000 \quad R=1.9 \\
& 0.49 \cdot\left(\pi_{P} \rightarrow \pi_{M}{ }^{*}\right)-0.49 \cdot\left(\pi_{M} \rightarrow \pi_{P}{ }^{*}\right) \\
& \mathbf{S}_{0} \rightarrow \mathbf{S}_{2} \quad f=0.000 \quad R=-0.2 \\
& 0.49 \cdot\left(\pi_{P} \rightarrow \pi_{M}{ }^{*}\right)+0.49 \cdot\left(\pi_{M} \rightarrow \pi_{P}{ }^{*}\right) \\
& \mathbf{S}_{0} \rightarrow \mathbf{S}_{1} \quad f=0.000 \quad R=2.3 \\
& 0.49 \cdot\left(\pi_{M} \rightarrow \pi_{M}{ }^{*}\right)+0.49 \cdot\left(\pi_{P} \rightarrow \pi_{P}{ }^{*}\right)
\end{aligned}
$$

Figure 3. a) Overview of first four electronic transitions of allene and $R$-1,3-dimethylallene, and their symmetry designations. Electric-dipole allowed transitions are marked in blue. b) Change of electron density for the $\mathrm{S}_{0} \rightarrow \mathrm{S}_{1}, \mathrm{~S}_{0} \rightarrow \mathrm{S}_{2}, \mathrm{~S}_{0} \rightarrow \mathrm{S}_{3}$ and $\mathrm{S}_{0} \rightarrow \mathrm{S}_{4}$ electronic transitions of $S$ 1,4-dimethylallene computed at the $\omega$ B97X-D/Def2-TZVP level of theory. ${ }^{45}, 46$ Oscillator strengths, $f$, are dimensionless. Rotatory strengths, $R$, are in $10^{40} \mathrm{erg} \cdot \mathrm{esu} \cdot \mathrm{cm} /$ Gauss. Depletion of density is in orange. Iso-value $=0.002$.

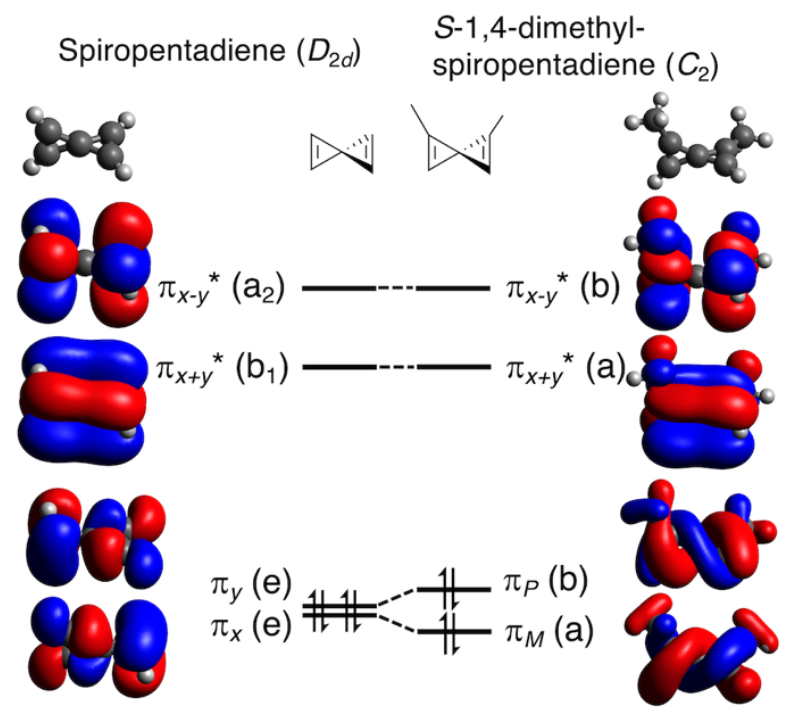

Figure 4. HOMO-1 to LUMO+1 of spiropentadiene and $S$-1,4-dimethylspiropentadiene computed at the $\omega$ B97X-D/Def2-TZVP level of theory. ${ }^{45,46}$

In $D_{2 d}$-spiropentadiene, $\pi_{\mathrm{y}}$ and $\pi_{x}$ are degenerate while $\pi_{x+y} *$ and $\pi_{\mathrm{x}-\mathrm{y}} *$ are non-degenerate. Consequently, the four $\pi \rightarrow \pi^{*}$ transitions will be two degenerate pairs belonging to the E representation as verified by the direct products of the $\mathrm{E}$ with $\mathrm{A}_{2}$ and $B_{1}$ representations in Figure 5a. ${ }^{38,57,58}$ All four $\pi \rightarrow \pi^{*}$ transitions are electric-dipole allowed, and this does not change when the symmetry is reduced by substituents. In dimethylspiropentadiene the electronic transitions become non-degenerate, but do not split by much energetically. Different from dimethylallene, the transitions are not equally weighted linear combinations. Take the two lowest energy MO excitations; $\pi_{P} \rightarrow \pi_{x+y} *$ belong to the $\mathrm{B}$ irreducible representation while $\pi_{M} \rightarrow \pi_{x+y} *$ belong to the $\mathrm{A}$ irreducible representation $(\mathrm{A} \times \mathrm{A}=$ A). Because they are symmetry-protected, these transitions cannot mix and do not form a superposition. As listed in Figure 5b, there is configuration interaction with higher energy excitations of same symmetry. However, excitations into $\pi_{\mathrm{x}-\mathrm{y}}$ * contribute less than $10 \%$ to the $\mathrm{S}_{0} \rightarrow \mathrm{S}_{1}$ and $\mathrm{S}_{0} \rightarrow \mathrm{S}_{2}$ transitions. A qualitatively similar result is achieved at the CASPT2 $(8,12) / \mathrm{cc}-$ pvdz level (Figure S2).

All four $\pi-\pi^{*}$ transitions have clear helicity associated to the change of electron density as shown in Figure 5b. We also verify schematically in Figure S5 that all excitations from a helical to a linear MO will yield helical change of electron density. The through-bond interaction of the two $\pi$-systems mediated by the spiro-carbon thus manifests itself in the electron density. The first four electronic transitions all have notable rotatory strengths, in particular the strong $\mathrm{S}_{0} \rightarrow \mathrm{S}_{3}$ and $\mathrm{S}_{0} \rightarrow \mathrm{S}_{4}$ transitions. Each pair of near-degenerate transitions consist of opposite helicity in the change of electron density, and have opposite sign of the rotatory strength. Circularly polarized light will thus be more likely to absorb due to transitions of a specific helicity. This underlines the connection between the helicity of the electronic structure and the chiroptical response of the molecule. It is possible that a helical change of electron density and the rotatory strength of a transition are fundamentally related. 


$$
\begin{aligned}
& D \times 1 \\
& \mu_{x, y, z}=E, E, B_{2} \\
& E \times A_{2}=E \\
& E \times B_{1}=E
\end{aligned}
$$

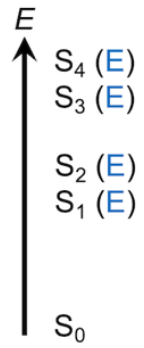

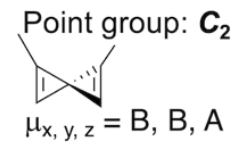

$A \times A=A$

$A \times B=B$

$$
B \times B=A
$$

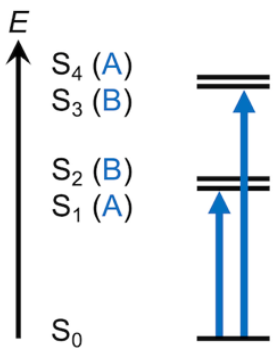

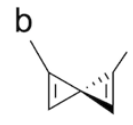

$$
\mathbf{S}_{0} \rightarrow \mathbf{S}_{4} \quad f=0.068 \quad R=216.0
$$$$
0.67 \cdot\left(\pi_{P} \rightarrow \pi_{\mathrm{x}-\mathrm{y}}{ }^{*}\right)+0.18 \cdot\left(\pi_{M} \rightarrow \pi_{\mathrm{x}+\mathrm{y}}{ }^{*}\right)
$$

$$
\mathbf{S}_{0} \rightarrow \mathbf{S}_{3} f=0.071 \quad R=-217.9
$$$$
0.67 \cdot\left(\pi_{M} \rightarrow \pi_{\mathrm{x}-\mathrm{y}}{ }^{*}\right)+0.18 \cdot\left(\pi_{P} \rightarrow \pi_{\mathrm{x}+\mathrm{y}}{ }^{*}\right)
$$$$
\mathbf{S}_{0} \rightarrow \mathbf{S}_{2} \quad f=0.012 \quad R=16.1
$$$$
0.67 \cdot\left(\pi_{P} \rightarrow \pi_{\mathrm{x}+\mathrm{y}}{ }^{*}\right)-0.18 \cdot\left(\pi_{M} \rightarrow \pi_{\mathrm{x}-\mathrm{y}}{ }^{*}\right)
$$$$
\mathbf{S}_{0} \rightarrow \mathbf{S}_{1} \quad f=0.012 \quad R=-16.3
$$$$
0.67 \cdot\left(\pi_{M} \rightarrow \pi_{\mathrm{x}+\mathrm{y}}{ }^{*}\right)-0.18 \cdot\left(\pi_{P} \rightarrow \pi_{\mathrm{x}-\mathrm{y}}{ }^{*}\right)
$$
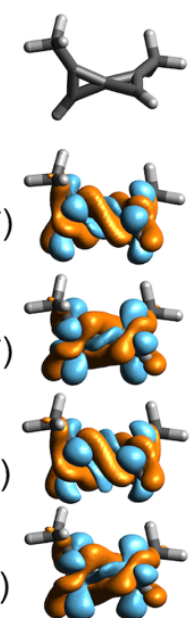

Figure 5. a) Overview of first four electronic transitions of spiropentadiene and $S$-1,4-dimethylspiropentadiene, and their symmetry designations. Electric-dipole allowed transitions are marked in blue. b) Change of electron density for the $\mathrm{S}_{0} \rightarrow \mathrm{S}_{1}, \mathrm{~S}_{0} \rightarrow \mathrm{S}_{2}, \mathrm{~S}_{0} \rightarrow \mathrm{S}_{3}$ and $\mathrm{S}_{0} \rightarrow \mathrm{S}_{4}$ electronic transitions of S-1,4-dimethylspiropentadiene computed at the $\omega$ B97X-D/Def2-TZVP level of theory. ${ }^{45,}{ }^{46}$ Oscillator strengths, $f$, are dimensionless. Rotatory strengths, $R$, are in $10^{40} \mathrm{erg} \cdot \mathrm{esu} \cdot \mathrm{cm} /$ Gauss. Depletion of density is in orange. Iso-value $=0.002$.

The overall optical activity of dimethylspiropentadiene will be small unless the near-degenerate transitions can be split energetically, as opposite sign in the rotatory strengths cancels the overall chiroptical response of the molecule. The substituent strategy we used to split the helical frontier MOs of allene and longer odd-carbon cumulenes appears to be ineffective in spiropentadiene and larger spiroconjugated molecules. ${ }^{20} \mathrm{We}$ instead explore strained allenes and spiropentadienes by applying a cyclically-connected butadiyl-substiuent to the molecules as illustrated for spiropentadiene in Figure 6. This alters the energetics of the frontier MOs considerably as the dihedral and bond angles of the allenic and spiro-segments are strained, thereby unwinding or overwinding the helices. ${ }^{3,17}$ While both molecules show increased chiroptical response, cf. Figure S6 and S7, the strained $R$-1,4-butadiylspiropentadiene sees a major change compared to the dimethylspiropentadiene as shown in the simulated electronic circular dichroism (ECD) spectrum in Figure 6 . The big rotatory strengths of the $\mathrm{S}_{0} \rightarrow \mathrm{S}_{3}$ and $\mathrm{S}_{0} \rightarrow \mathrm{S}_{4}$ transitions now give rise to two opposite peaks in the simulated ECD spectrum. It is likely that the extra strain in these species will destabilize them. We include them as a computational proof-ofconcept for how the electronic structure of spiroconjugated molecules can be manipulated to maximize the chiroptical response.

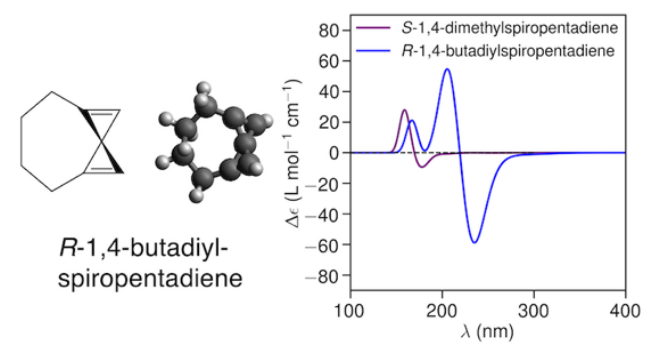

Figure 6. Simulated ECD spectrum of dimethylspiropentadiene and butadiyl-linked spiropentadiene computed at the $\omega \mathrm{B} 97 \mathrm{X}-\mathrm{D} / \mathrm{Def} 2-$ TZVP level of theory. ${ }^{45,46}$

The detailed description we have provided of spiropentadiene is in principle valid for larger spiroconjugated motives and tricyclic analogues, such as those shown in scheme $2 .{ }^{58-60} \mathrm{In}$ larger spiroconjugated systems, such as spirononatetraene, there are more $\pi$-electrons and therefore the MO symmetries switch. Furthermore, the helicity is less obvious in higher energy MOs due to the sharper twist. The HOMO-2 and HOMO3 of dimethylspirononatetraene are clearly helical (Figure S8), but will be less relevant for observable properties. In tricyclic spiro-analogues where the parent system has $D_{2 d}$ symmetry will also have similar mixing of its two $\pi$-systems; albeit there will be extra nodal planes due to the extra $\sigma$-bonds (Figure S10 and $\mathrm{S} 11 .^{30,31,61}$ Helical $\pi$-mixing in larger spiroconjugated motives will be subject to further investigations.

\section{Scheme 2. Larger D2d-symmetry molecules.}
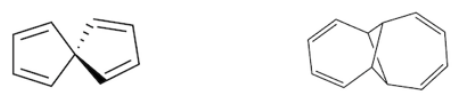

Spirononatetraene Tricyclododecatetraene

In summary, We have explored how helical $\pi$-orbitals manifest themselves in the electronic structure of allene, spiropentadiene, and spirononatetraene. In the electronic transitions of allene the helicity of the $\pi$-MOs cancel out as all transitions are linear combinations of excitations involving MOs of both helicities. In spiropentadiene, the HOMO-1 and HOMO become helical in similar fashion to the $\pi$-mixing in allene, but the LUMO and LUMO+1 remain rectilinear $\pi$-MOs. The $\pi \rightarrow \pi^{*}$ transitions are dominated by a single configuration, which is symmetry-protected from mixing with excitations of opposite helicity. These transitions have a clear helicity associated to the change of electron density, and the rotatory strength of each transition has opposite sign from the near-degenerate transition of opposite helicity. The electrohelicity effect in spiropentadiene thus manifests itself in the optical properties of the molecule. Our results underline the continued need for improving the chemical intuition for chiroptical properties of molecules. ${ }^{62} \mathrm{Fi}$ nally, these results suggest that helical MOs can manifest in observable properties, and, as has been noted elsewhere, ${ }^{63}$ an understanding of the electrohelicity effect beyond mean-field theory is desired. 


\section{ASSOCIATED CONTENT}

\section{Supporting Information}

Computational Details. CASPT2 computations, Change of density schematics, Cyclically-linked molecules, Spirononatetraene, Tricyclic spiro-analogues.

\section{Corresponding Author}

* E-mail: clemence.corminboeuf@epfl.ch.

\section{Notes}

The authors declare no competing financial interest.

\section{ACKNOWLEDGMENT}

The authors thank Maria Fumanal (Ecole Polytechnique Federale de Lausanne) for technical help with CASPT2 computations. MHG is grateful for funding from Independent Research Fund Denmark, case no. 9056-00009B

\section{REFERENCES}

1. Frenking, G.; Krapp, A., Unicorns in the world of chemical bonding models. J. Comp. Chem. 2007, 28, 15-24.

2. Hendon, C. H.; Tiana, D.; Murray, A. T.; Carbery, D. R.; Walsh, A., Helical frontier orbitals of conjugated linear molecules. Chem. Sci. 2013, 4, 4278-4284.

3. Imamura, A.; Aoki, Y., Helical molecular orbitals around straight-chain polyyne oligomers as models for molecular devices. Chem. Phys. Lett. 2013, 590, 136-140.

4. Garner, M. H.; Hoffmann, R.; Rettrup, S.; Solomon, G. C., Coarctate and Möbius: The Helical Orbitals of Allene and Other Cumulenes. ACS Cent. Sci. 2018, 4, 688-700.

5. Krylov, A. I., From orbitals to observables and back. $J$ Chem. Phys. 2020, 153, 080901.

6. Woodward, R. B.; Hoffmann, R., The Conservation of Orbital Symmetry. Angew. Chem. Int. Ed. Engl. 1969, 8, 781-853.

7. Fukui, K., Recognition of stereochemical paths by orbital interaction. Acc. Chem. Res. 1971, 4, 57-64.

8. Zimmerman, H. E., Moebius-Hueckel concept in organic chemistry. Application of organic molecules and reactions. Acc. Chem. Res. 1971, 4, 272-280.

9. Houk, K. N., Frontier molecular orbital theory of cycloaddition reactions. Acc. Chem. Res. 1975, 8, 361-369.

10. Xu, T.; Li, J. H.; Momen, R.; Huang, W. J.; Kirk, S. R.; Shigeta, Y.; Jenkins, S., Chirality-Helicity Equivalence in the S and R Stereoisomers: A Theoretical Insight. J. Am. Chem. Soc. 2019, 141, 5497-5503.

11. Tiana, D.; Hendon, C. H.; Walsh, A., Ligand design for long-range magnetic order in metal-organic frameworks. Chem. Comm. 2014, 50, 13990-13993.

12. Caricato, M., Orbital Analysis of Molecular Optical Activity Based on Configuration Rotatory Strength. J. Chem. Theory Comput. 2015, 11, 1349-1353.

13. Peeks, M. D.; Neuhaus, P.; Anderson, H. L., Experimental and computational evaluation of the barrier to torsional rotation in a butadiyne-linked porphyrin dimer. Phys. Chem. Chem. Phys. 2016, 18 , 5264-5274.

14. Martin, W. R.; Ball, D. W., Small Organic Azides as High Energy Materials: Perazidoacetylene, -ethylene, and -allene. ChemistrySelect 2018, 3, 7222-7225.

15. Garner, M. H.; Jensen, A.; Hyllested, L. O. H.; Solomon, G. C., Helical Orbitals and Circular Currents in Linear Carbon Wires. Chem. Sci. 2019, 10, 4598-4608.

16. Ozcelik, A.; Aranda, D.; Gil-Guerrero, S.; Pola-Otero, X. A.; Talavera, M.; Wang, L.; Behera, S. K.; Gierschner, J.; Peña-Gallego, Á.; Santoro, F.; Pereira-Cameselle, R.; Alonso Gómez, J. L., Distinct helical molecular orbitals through conformational lock. Chem. Eur. J. 2020, 26, 17342-17349.
17. Garner, M. H.; Bro-Jørgensen, W.; Solomon, G. C., Three Distinct Torsion Profiles of Electronic Transmission through Linear Carbon Wires. J. Phys. Chem. C 2020, 124, 18968-18982.

18. Gunasekaran, S.; Venkataraman, L., Tight-binding analysis of helical states in carbyne. J. Chem. Phys. 2020, 153, 124304.

19. Pinter, P.; Munz, D., Controlling Möbius-Type Helicity and the Excited-State Properties of Cumulenes with Carbenes. J. Phys. Chem. A 2020, 124, 10100-10110.

20. Garner, M. H.; Corminboeuf, C., Correlation between Optical Activity and the Helical Molecular Orbitals of Allene and Cumulenes. Org. Lett. 2020, 22, 8028-8033.

21. Fischer, H.; Kollmar, H., Zur Invarianz in der LCAO MO Theorie. Theor. Chim. Acta 1968, 12, 344-348.

22. Herges, R., Organizing Principle of Complex Reactions and Theory of Coarctate Transition States. Angew. Chem. Int. Ed. 1994, 33, 255-276.

23. Herges, R., Coarctate and Pseudocoarctate Reactions: Stereochemical Rules. J. Org. Chem. 2015, 80, 11869-11876.

24. Simmons, H. E.; Fukunaga, T., Spiroconjugation. J. Am. Chem. Soc. 1967, 89, 5208-5215.

25. Hoffmann, R.; Imamura, A.; Zeiss, G. D., Spirarenes. J. Am. Chem. Soc. 1967, 89, 5215-5220.

26. Gleiter, R.; Schaefer, W., Interactions between nonconjugated .pi.-systems. Acc. Chem. Res. 1990, 23, 369-375.

27. Böhm, M. C.; Gleiter, R., Model calculations on spirocompounds with silicon and phosphorus as central atoms. J. Chem. Soc., Perkin Trans. 2 1979, 443-448.

28. de Meijere, A.; Khlebnikov, A. F.; Kostikov, R. R.; Kozhushkov, S. I.; Schreiner, P. R.; Wittkopp, A.; Yufit, D. S., The First Enantiomerically Pure Triangulane (M)Trispiro[2.0.0.2.1.1]nonane Is a $\sigma-[4]$ Helicene. Angew. Chem. Int. Ed. 1999, 38, 3474-3477.

29. de Meijere, A.; Kozhushkov, S. I.; Fokin, A. A.; Emme, I.; Redlich, S.; Schreiner, P. R., New structurally interesting cyclopropane derivatives. A world of wonders and surprises. Pure Appl. Chem. 2003, $75,549-562$.

30. Bischof, P.; Gleiter, R.; Haider, R., Through-bond interaction of two mutually perpendicular .pi. systems. A comparison with spiroconjugation. J. Am. Chem. Soc. 1978, 100, 1036-1042.

31. Gleiter, R.; Haider, R.; Spanget-Larsen, J.; Bischof, P., Evidence for through-bond interaction between mutually perpendicular PI systems. Tetrahedron Lett. 1983, 24, 1149-1152.

32. M. J. Frisch, G. W. T., H. B. Schlegel, G. E. Scuseria, M. A. Robb, J. R. Cheeseman, G. Scalmani, V. Barone, G. A. Petersson, H. Nakatsuji, X. Li, M. Caricato, A. V. Marenich, J. Bloino, B. G. Janesko, R. Gomperts, B. Mennucci, H. P. Hratchian, J. V. Ortiz, A. F Izmaylov, J. L. Sonnenberg, D. Williams-Young, F. Ding, F. Lipparini, F. Egidi, J. Goings, B. Peng, A. Petrone, T. Henderson, D. Ranasinghe, V. G. Zakrzewski, J. Gao, N. Rega, G. Zheng, W. Liang, M. Hada, M. Ehara, K. Toyota, R. Fukuda, J. Hasegawa, M. Ishida, T. Nakajima, Y. Honda, O. Kitao, H. Nakai, T. Vreven, K. Throssell, J. A. Montgomery, Jr., J. E. Peralta, F. Ogliaro, M. J. Bearpark, J. J. Heyd, E. N. Brothers, K. N. Kudin, V. N. Staroverov, T. A. Keith, R. Kobayashi, J. Normand, K. Raghavachari, A. P. Rendell, J. C. Burant, S. S. Iyengar, J. Tomasi, M. Cossi, J. M. Millam, M. Klene, C. Adamo, R. Cammi, J. W. Ochterski, R. L. Martin, K. Morokuma, O. Farkas, J. B. Foresman, and D. J. Fox Gaussian 16, Revision A.03, Gaussian, Inc., Wallingford CT2016.

33. Stephens, P. J.; Devlin, F. J.; Cheeseman, J. R.; Frisch, M. J., Calculation of Optical Rotation Using Density Functional Theory. J. Phys. Chem. A 2001, 105, 5356-5371.

34. Autschbach, J.; Ziegler, T.; van Gisbergen, S. J. A.; Baerends, E. J., Chiroptical properties from time-dependent density functional theory. I. Circular dichroism spectra of organic molecules. J. Chem. Phys. 2002, 116, 6930-6940.

35. Srebro-Hooper, M.; Autschbach, J., Calculating Natural Optical Activity of Molecules from First Principles. Annu. Rev. Phys. Chem. 2017, 68, 399-420.

36. Soriano, E.; Fernandez, I., Allenes and computational chemistry: from bonding situations to reaction mechanisms. Chem. Soc. Rev. 2014, 43, 3041-3105. 
37. Wendinger, D.; Tykwinski, R. R., Odd [n]Cumulenes ( $\mathrm{n}=$ 3, 5, 7, 9): Synthesis, Characterization, and Reactivity. Acc. Chem. Res. 2017, 50, 1468-1479.

38. Cotton, F. A., Chemical Applications of Group Theory. 3rd ed.; John Wiley \& Sons, Inc.: New York, 1990.

39. Crabbe, P.; Velarde, E.; Anderson, H. W.; Clark, S. D.; Moore, W. R.; Drake, A. F.; Mason, S. F., Optical activity and absolute configuration of chiral allenes. J. Chem. Soc., Chem. Commun. 1971, 1261-1264.

40. Dickerson, H.; Ferber, S.; Richardson, F. S., Molecular orbital calculations on the optical rotatory properties of chiral allene systems. Theor. Chim. Acta 1976, 42, 333-344.

41. Rauk, A.; Drake, A. F.; Mason, S. F., Excited states and optical activity of allenes. Allene, 1,3-dimethylallene, and 1,2cyclononadiene. J. Am. Chem. Soc. 1979, 101, 2284-2289.

42. Elsevier, C. J.; Vermeer, P.; Gedanken, A.; Runge, W., Excited states of the allene chromophore: photoelectron, circular dichroism and absorption spectroscopy of alkyl- and halogenoallenes. J. Am. Chem. Soc. 1985, 107, 2537-2547.

43. Runge, W.; Baumann, H. F.; Hezemans, A. M. F.; Van de Coolwijk, P. J. F. M.; Elsevier, C. J.; Vermeer, P., The molecular structure of allenes and ketenes. XVIII. Excited states of the phenylallene chromophore and the role of doubly excited configurations in styrene-type molecules: circular dichroism and absorption spectra of 3-alkyl-1-phenylallenes and 1,3-diphenylallene. Chem. Phys. 1986, 105, 227-246.

44. Wiberg, K. B.; Wang, Y.-g.; Wilson, S. M.; Vaccaro, P. H.; Jorgensen, W. L.; Crawford, T. D.; Abrams, M. L.; Cheeseman, J. R.; Luderer, M., Optical Rotatory Dispersion of 2,3-Hexadiene and 2,3Pentadiene. J. Phys. Chem. A 2008, 112, 2415-2422.

45. Weigend, F.; Ahlrichs, R., Balanced basis sets of split valence, triple zeta valence and quadruple zeta valence quality for $\mathrm{H}$ to Rn: Design and assessment of accuracy. Phys. Chem. Chem. Phys. 2005, 7, 3297-3305.

46. Chai, J.-D.; Head-Gordon, M., Long-range corrected hybrid density functionals with damped atom-atom dispersion corrections. Phys. Chem. Chem. Phys. 2008, 10, 6615-6620.

47. Orimoto, Y.; Aoki, Y.; Imamura, A., Extraction of OneHanded Helical Frontier Orbital in Even [n]Cumulenes by Breaking Mirror Images of Right- and Left-Handed Helical Orbitals: Theoretical Study. J. Phys. Chem. C 2019, 123, 11134-11139.

48. Rzepa, H. S.; Taylor, K. R., Möbius and Hückel spiroaromatic systems. J. Chem. Soc., Perkin Trans. 2 2002, 14991501 .

49. Hall, D.; Rzepa, H. S., Möbius bis and tris-spiroaromatic systems. Org. Biomol. Chem. 2003, 1, 182-185.

50. Zhang, Y.; Wei, J.; Chi, Y.; Zhang, X.; Zhang, W.-X.; Xi, Z., Spiro Metalla-aromatics of $\mathrm{Pd}, \mathrm{Pt}$, and $\mathrm{Rh}$ : Synthesis and Characterization. J. Am. Chem. Soc. 2017, 139, 5039-5042.

51. Kenny, J. P.; Krueger, K. M.; Rienstra-Kiracofe, J. C.; Schaefer, H. F., C5H4: Pyramidane and Its Low-Lying Isomers. J. Phys. Chem. A 2001, 105, 7745-7750.

52. Billups, W. E.; Haley, M. M., Spiropentadiene. J. Am. Chem. Soc. 1991, 113, 5084-5085.

53. Billups, W. E.; McCord, D. J., Gas-Phase Synthesis of Reactive Molecules Using Adsorbed Reagents. Angew. Chem. Int. Ed. Engl. 1994, 33, 1332-1343.

54. Saini, R. K.; Litosh, V. A.; Daniels, A. D.; Billups, W. E., Synthesis and characterization of 1,4-dichlorospiropentadiene. Tetrahedron Lett. 1999, 40, 6157-6158.

55. Iwamoto, T.; Tamura, M.; Kabuto, C.; Kira, M., A Stable Bicyclic Compound with Two $\mathrm{Si}=\mathrm{Si}$ Double Bonds. Science 2000, 290, 504 .

56. Guo, Y.; Xia, Z.; Liu, J.; Yu, J.; Yao, S.; Shi, W.; Hu, K.; Chen, S.; Wang, Y.; Li, A.; Driess, M.; Wang, W., A Tetra-amidoProtected Ge5-Spiropentadiene. J. Am. Chem. Soc. 2019, 141, $19252-$ 19256.
57. Batich, C.; Heilbronner, E.; Rommel, E.; Semmelhack, M. F.; Foos, J. S., Equivalence of the energy gaps .DELTA.I $(1,2)$ and .DELTA.E $(1,2)$ between corresponding bands in the photoelectron (I) and electronic absorption (E) spectra of spiro[4.4]nonatetraene. Amusing consequence of spiroconjugation. J. Am. Chem. Soc. 1974, $96,7662-7668$.

58. Dürr, H.; Gleiter, R., Spiroconjugation. Angew. Chem. Int. Ed. Engl. 1978, 17, 559-569.

59. Haselbach, E.; Allan, M.; Bally, T.; Bednarek, P.; Sergenton, A.-C.; de Meijere, A.; Kozhushkov, S.; Piacenza, M.; Grimme, S., Spiro[4.4]nonatetraene and its Positive and Negative Radical Ions: Molectronic Structure Investigations. Helv. Chim. Acta 2001, 84, 1670 1693.

60. Sowa, J. K.; Mol, J. A.; Briggs, G. A. D.; Gauger, E. M., Spiro-Conjugated Molecular Junctions: Between Jahn-Teller Distortion and Destructive Quantum Interference. J. Phys. Chem. Lett. 2018, 9, 1859-1865.

61. Dressel, J.; Chasey, K. L.; Paquette, L. A., Through-bond interaction via cyclobutane relay orbitals as a means of extending conjugation. Synthesis of tricyclo[5.5.0.02,8]dodecatetraene, tricyclo[5.3.0.02,8]deca-3,5,9-triene, and 9,10dimethylenetricyclo[5.3.0.02,8]deca-3,5-diene. J. Am. Chem. Soc. 1988, $110,5479-5489$.

62. de Wergifosse, M.; Grimme, S., A Unified Strategy for the Chemically Intuitive Interpretation of Molecular Optical Response Properties. J. Chem. Theory Comput. 2020, 16, 7709-7720.

63. Eriksen, J. J., Mean-field density matrix decompositions. J. Chem. Phys. 2020, 153, 214109. 